\title{
Adubação com potássio e cálcio na nutrição e produção de goiabeira 'Paluma'
}

\author{
Alian Cássio Pereira Cavalcante ${ }^{2 *}\left(\mathbb{D}\right.$, Lourival Ferreira Cavalcante ${ }^{3}$, Antonio Michael Pereira Bertino ${ }^{4}$, \\ Adailza Guilherme Cavalcante ${ }^{4}$, Antonio João de Lima Neto ${ }^{2}$, Nubia Marisa Ferreira ${ }^{5}$
}

10.1590/0034-737X201966010008

\section{RESUMO}

A goiabeira é uma frutífera cultivada mundialmente, destacando-se pela grande aceitação no mercado consumidor. Entretanto, para que a cultura explore o seu máximo potencial produtivo e mantenha a qualidade dos frutos, é necessária a adequada adubação mineral para elevar a disponibilidade de nutrientes no solo e aumentar sua absorção pelas plantas. Objetivou-se, com este trabalho, avaliar os efeitos da adubação com doses de potássio, na presença e na ausência de cálcio, no estado nutricional e na produção da goiabeira 'Paluma'. $\mathrm{O}$ experimento foi desenvolvido de agosto de 2015 a maio de 2016, no município de Remígio, Paraíba. As variáveis analisadas foram: teores foliares de macro e de micronutrientes, número de frutos por planta, massa média de frutos, produção de frutos por planta e produtividade. As plantas da goiabeira estavam adequadamente supridas em nitrogênio, fósforo, potássio, cálcio, magnésio, boro, ferro, manganês e deficientes em enxofre, cobre e zinco. A adubação com cálcio incrementou os teores foliares de $\mathrm{P}, \mathrm{K}, \mathrm{Ca}, \mathrm{S}, \mathrm{B}, \mathrm{Mn}$ e Zn e reduziu o número de frutos por planta e a produtividade da goiabeira 'Paluma'. A adubação potássica sem adição de cálcio, nas doses entre 101 e $143 \mathrm{~g} \mathrm{planta}^{-1}$ ano $^{-1}$ de $\mathrm{K}_{2} \mathrm{O}$, aos 16 meses de idade, proporcionou a maior produtividade da goiabeira 'Paluma'.

Palavras-chave: Psidium guajava L.; adubação; nutrição; produtividade.

\section{ABSTRACT}

\section{Fertilization with potassium and calcium in nutrition and production of guava 'Paluma'}

The guava is a fruit cultivated worldwide, distinguished by the great acceptance in the consumer market. However, so that the crop exploits its maximum productive potential and maintains the quality of the fruits it is necessary an adequate mineral fertilization to raise the availability of nutrients in the soil and to increase the absorption by plants. The objective of the work was to evaluate the effects of fertilization with potassium doses, in the presence and absence of calcium, on the nutritional status and on the production of 'Paluma' guava. The experiment was carried out from August 2015 to May 2016, in the municipality of Remígio-PB, Brazil. The variables analyzed were: macro and micronutrient leaf contents, number of fruits per plant, average fruit mass, fruit yield per plant and productivity. Guava plants were adequately supplied with nitrogen, phosphorus, potassium, calcium, magnesium, boron, iron and manganese, and deficient in sulfur, copper and zinc. Calcium fertilization increased the leaf content of $\mathrm{P}, \mathrm{K}, \mathrm{Ca}, \mathrm{S}, \mathrm{B}, \mathrm{Mn}$ and $\mathrm{Zn}$, but reduced the number of fruits per plant and the yield of 'Paluma' guava. Potassium fertilization without calcium addition at doses between 101 and $143 \mathrm{~g} \mathrm{plant}^{-1}$ year ${ }^{-1} \mathrm{~K}_{2} \mathrm{O}$ at 16 months of age provides the highest productivity of 'Paluma' guava.

Keywords: Psidium guajava L.; fertilizing; nutrition; productivity.

\footnotetext{
Submetido em 20/03/2018 e aprovado em 05/02/2019

${ }^{1}$ Este trabalho faz parte da dissertação do primeiro autor.

${ }^{2}$ Universidade Federal de Viçosa, Programa de Pós-Graduação em Fitotecnia, Viçosa, Minas Gerais, Brasil. cassio.alian216@gmail.com; limanetoagro@hotmail.com

${ }^{3}$ Universidade Federal da Paraíba, Programa de Pós-Graduação em Agronomia da Universidade Federal da Paraíba, Areia, Paraíba, Brasil. lofeca1946@yahoo.com.br

${ }^{4}$ Universidade Estadual Paulista, Programa de Pós-Graduação em Agronomia, Jaboticabal, São Paulo, Brasil. adailzacavalcante@gmail.com; ampbantonio@gmail.com

${ }_{5}^{5}$ Universidade Federal Rural do Semi-Árido, Programa de Pós-Graduação em Fitotecnia, Mossoró, Rio Grande do Norte, Brasil. nubiamarisa1@hotmail.com

"Autor para correspondência: cassio.alian216@gmail.com
}

Rev. Ceres, Viçosa, v. 66, n.1, p. 054-062, jan/fev, 2019 


\section{INTRODUÇÃO}

A goiabeira (Psidium guajava L.) é uma frutífera tropical, originária das Américas do Sul e Central, que pode ser encontrada em todo o território brasileiro. Seus frutos apresentam sabor agradável, elevado valor nutricional e boa aceitação pelos consumidores, podendo ser consumidos in natura ou utilizados nas agroindústrias de processamento (Montes et al., 2016).

A goiabeira é muito exigente em potássio, sendo este o nutriente mais exportado pelos frutos (Natale et al., 2009b; Souza et al., 2012). O cálcio, apesar de exportado em menores quantidades, melhora a qualidade do fruto, contribui para a maior firmeza da casca e reduz as perdas após a colheita (Natale et al., 2005), o que torna imprescindível a fertilização da goiabeira com esses nutrientes.

A adequada adubação mineral da goiabeira é uma prática indispensável para garantir produtividades elevadas; enquanto a avaliação do estado nutricional das plantas contribui para melhorar a eficiência das fertilizações (Amorim et al., 2015b). Esses autores, ao avaliarem a adubação potássica sobre o estado nutricional de plantas de goiabeira 'Paluma', constataram aumento dos teores foliares de K, sem influência na produtividade dessa frutífera (Amorim et al., 2015a).

Os solos brasileiros, em geral, apresentam baixa fertilidade, por causa do cultivo contínuo, tornando-se necessárias as adubações para o crescimento e produção das culturas. Por essa razão, pesquisas vêm buscando informações sobre fontes, doses e melhor forma de aplicação dos nutrientes e a resposta em produção das culturas (Silva et al., 2001; França et al., 2017).

A adubação adequada é crucial para se elevar a disponibilidade de nutrientes no solo e aumentar sua absorção pelas plantas de forma equilibrada (Aular \& Natale, 2013). Além de proporcionar menor susceptibilidade das plantas ao ataque de pragas e doenças, maior tolerância a períodos de seca e a outros estresses, a adubação contribui para aumentar a produtividade da cultura e melhorar a qualidade dos frutos (Dias et al., 2012).

O potássio desempenha um papel crucial em vários processos fisiológicos que alteram o crescimento e, consequentemente, a produtividade das culturas. Esse nutriente atua na regulação da abertura estomática, limitando a perda de água, bem como nos processos fotossintéticos das plantas (Zorb et al., 2014; Cavalcante et al., 2018). Outro nutriente de grande relevância é o cálcio, por sua contribuição ao estímulo do crescimento e desenvolvimento das plantas, com incremento da massa dos frutos, por melhoria da estrutura da parede celular, além de estimulação do crescimento radicular (Karley \& White, 2009; Kumar et al., 2017).
Embora os nutrientes exigidos para a adequada nutrição das plantas sejam os mesmos para todos os vegetais, é necessário determinar as doses que proporcionam maior produção e eficiência econômica no pomar de goiabeira (Alencar et al., 2016). Diante da importância de determinar as necessidades nutricionais da goiabeira, este trabalho objetivou avaliar os efeitos da presença e da ausência de cálcio e das doses de potássio no estado nutricional das plantas e na produção de frutos da goiabeira 'Paluma'.

\section{MATERIAL E MÉTODOS}

O experimento foi realizado na propriedade Sítio Macaquinhos, município de Remígio, Estado da Paraíba, de agosto de 2015 a maio de 2016 . O municipio está localizado a 653'00" S, 3602'00" O, a 470 m acima do nível do mar. Pela classificação de Köppen (Alvares et al. , 2013), o clima é do tipo As', que significa quente e úmido, com chuvas concentradas de março a julho.

O solo da área experimental foi classificado como Neossolo Regolítico, conforme os critérios do Sistema Brasileiro de Classificação de Solos - SiBCS (Embrapa, 2013). Antes da instalação do experimento foram coletadas amostras de solo na camada de 0-40 cm, na área de projeção da copa das plantas, para caracterização química quanto à fertilidade e dos atributos físicos (Tabela 1), adotando-se as metodologias sugeridas por Embrapa (2011).

O pomar de goiabeiras do cultivar 'Paluma' foi instalado em julho de 2014, com mudas propagadas por estaquia e, aos 13 meses após o plantio, foram aplicados os tratamentos. As plantas foram cultivadas no espaçamento de $3 \times 3 \mathrm{~m}$ e, para redução das perdas de água por evaporação, as áreas de projeção das copas foram mantidas cobertas com uma camada de capim Brachiaria decumbens de $8 \mathrm{~cm}$ de espessura, que foi avaliada, quanto às características químicas, antes e ao final do experimento (Tabela 1).

$\mathrm{O}$ delineamento experimental utilizado foi em blocos casualizados, no arranjo fatorial $2 \times 5$, referente ao solo sem e com cálcio e cinco doses de potássio, com três repetições e três plantas por parcela. O cálcio foi fornecido na dose de $120 \mathrm{~g}_{\text {planta }}{ }^{-1}$ ano $^{-1}$, utilizando-se como fonte o nitrato de cálcio ( $19,0 \%$ de Ca e 15,5\% de N), 15 dias antes das adubações com NPK. O potássio foi fornecido nas doses de $0,45,90,135$ e 180 g planta $^{-1}$ ano $^{-1}$ de $\mathrm{K}_{2} \mathrm{O}$, na forma de cloreto de potássio $\left(56 \%\right.$ de $\left.\mathrm{K}_{2} \mathrm{O}\right)$.

As plantas de goiabeira foram adubadas com $160 \mathrm{~g}$ planta $^{-1}$ ano $^{-1}$ de N, tendo como fonte a ureia $(45 \%$ de N) e $120 \mathrm{~g} \mathrm{planta}^{-1}$ ano $^{-1}$ de $\mathrm{P}_{2} \mathrm{O}_{5}$, utilizando-se o fosfato monoamônico (MAP) como fonte ( $49 \%$ de $\mathrm{P}_{2} \mathrm{O}_{5}$ e $10 \%$ de $\mathrm{N})$, seguindo-se as recomendações de Natale et al. (2009a). 
Essas doses foram parceladas em três vezes e aplicadas na área de projeção da copa, sendo que, para o N, $\mathrm{K}_{2} \mathrm{O}$ e $\mathrm{Ca}, 30 \%$ foram aplicados após a poda das plantas; $30 \%$, no início da floração e, $40 \%$, aos 30 dias após o pleno florescimento. Para o fósforo, as proporções foram de 50, 25 e $25 \%$, aplicadas no mesmo período. No caso da dose de $\mathrm{N}$, foi descontada a quantidade do nutriente fornecida pelo nitrato de cálcio e pelo MAP.

Foram aplicados ainda, em cobertura, juntamente com a primeira adubação de NPK, $20 \mathrm{~g} \mathrm{cova}^{-1}$ de sulfato de zinco (20\% de $\mathrm{Zn}$ ) e $30 \mathrm{~g} \mathrm{cova}^{-1}$ de sulfato de magnésio (9\% de $\mathrm{Mg}$ ), em virtude dos baixos teores desses elementos no solo (Tabela 1). Foram realizadas também três pulverizações por via foliar com bórax (11\% de B), conforme sugestão de Natale et al. (2009a).

A irrigação das plantas foi feita por sistema de irrigação por microaspersão, com eficiência de aplicação de $95 \%$ e eficiência de irrigação de $87,4 \%$, usando-se um emissor com vazão de $60 \mathrm{~L} \mathrm{~h}^{-1}$ por planta, instalado na altura para molhamento da área de projeção da copa das plantas, trabalhando na pressão de serviço de 0,2 MPa. A lâmina de água aplicada foi baseada na evapotranspiração potencial obtida, com base na evaporação de tanque classe 'A', instalado na área experimental.

O coeficiente de cultivo $(\mathrm{Kc})$ utilizado foi variável em função da fase fenológica das plantas: brotação e crescimento vegetativo $(0,68)$; período de floração e queda da fisiologia de frutos $(0,76)$; crescimento de frutos $(0,71)$; maturação e colheita de frutos $(0,62)$, seguindo-se recomendações de Teixeira et al. (2003a).

No período de pleno florescimento (90 dias após a primeira adubação e aos 16 meses de idade das plantas), foram coletadas amostras do terceiro par de folhas recémmaduras e com pecíolo, a partir do meristema apical dos ramos, nos quatro quadrantes das três plantas de cada parcela para avaliação do estado nutricional (Malavolta et al., 1997; Natale et al., 2009b).

Após a coleta foi feita a assepsia das folhas em água corrente e submersão em água deionizada. Esse material foi secado em estufa com circulação de ar forçado, a $65^{\circ} \mathrm{C}$, durante $72 \mathrm{~h}$, triturado em moinho tipo Willey TE - $650^{\circledR}$, utilizando-se peneira de 20 mesh, sendo, posteriormente, determinados os teores foliares de N, P, K, Ca, Mg, S, B, $\mathrm{Cu}, \mathrm{Fe}, \mathrm{Mn}$ e $\mathrm{Zn}$, empregando-se as metodologias sugeridas por Embrapa (2009).

Os frutos foram coletados semanalmente nas segundas, quartas e sextas-feiras, de janeiro a maio de 2016 e, após cada coleta, contados e pesados em balança analítica. Ao final da colheita, foram obtidos o número e a massa média de todos os frutos por planta, produção por planta e produtividade.

Os dados foram submetidos à análise de variância, as médias referentes à adubação sem e com cálcio foram comparadas pelo teste $\mathrm{F}$ a 1 e $5 \%$ de probabilidade e as doses de potássio foram comparadas por regressão, usando-se o software Statistical Analysis System SAS ${ }^{\circledR}$ (Cody, 2015).

Tabela 1: Atributos químicos e físicos do solo na camada de 0-40 cm, antes da instalação do experimento, e composição química da cobertura vegetal, antes da instalação do experimento e no final da produção

\begin{tabular}{|c|c|c|c|c|c|c|}
\hline \multirow{2}{*}{ Atributos Químicos } & \multirow{2}{*}{ Valor } & \multirow{2}{*}{ Atributos físicos } & \multirow{2}{*}{ Valor } & \multicolumn{3}{|c|}{ Composição química da cobertura vegetal } \\
\hline & & & & & Antes & Depois \\
\hline $\mathrm{pH}\left(\mathrm{H}_{2} \mathrm{O}\right)$ & 5,53 & Areia grossa $\left(\mathrm{g} \mathrm{kg}^{-1}\right)$ & 544 & $\mathrm{~N}\left(\mathrm{~g} \mathrm{~kg}^{-1}\right)$ & 11,9 & 18,0 \\
\hline $\mathrm{P}\left(\mathrm{mg} \mathrm{dm}^{-3}\right)$ & 9,7 & Areia fina $\left(\mathrm{g} \mathrm{kg}^{-1}\right)$ & 283 & $\mathrm{P}\left(\mathrm{g} \mathrm{kg}^{-1}\right)$ & 2,5 & 1,7 \\
\hline $\mathrm{K}^{+}\left(\mathrm{cmol}_{\mathrm{c}} \mathrm{dm}^{-3}\right)$ & 0,13 & Silte $\left(\mathrm{g} \mathrm{kg}^{-1}\right)$ & 91,0 & $\mathrm{~K}\left(\mathrm{~g} \mathrm{~kg}^{-1}\right)$ & 11,9 & 3,3 \\
\hline $\mathrm{Ca}^{2+}\left(\mathrm{cmol}_{\mathrm{c}} \mathrm{dm}^{-3}\right)$ & 1,79 & $\operatorname{Argila}\left(\mathrm{g} \mathrm{kg}^{-1}\right)$ & 83,0 & $\mathrm{Ca}\left(\mathrm{g} \mathrm{kg}^{-1}\right)$ & 4,6 & 12,9 \\
\hline $\mathrm{Mg}^{2+}\left(\mathrm{cmol}_{\mathrm{c}} \mathrm{dm}^{-3}\right)$ & 0,48 & Argila dispersa $\left(\mathrm{g} \mathrm{kg}^{-1}\right)$ & 19,0 & $\operatorname{Mg}\left(\mathrm{g} \mathrm{kg}^{-1}\right)$ & 2,8 & 2,6 \\
\hline $\mathrm{Na}^{+}\left(\mathrm{cmol}_{\mathrm{c}} \mathrm{dm}^{-3}\right)$ & 0,010 & Grau de floculação (\%) & 77,2 & $\mathrm{~S}\left(\mathrm{~g} \mathrm{~kg}^{-1}\right)$ & 2,0 & 1,6 \\
\hline $\mathrm{SB}\left(\mathrm{cmol}_{\mathrm{c}} \mathrm{dm}^{-3}\right)$ & 2,41 & Índice de dispersão (\%) & 28,2 & $\mathrm{Cu}\left(\mathrm{mg} \mathrm{kg}^{-1}\right)$ & 7,20 & 9,2 \\
\hline $\mathrm{H}^{+}+\mathrm{Al}^{3+}\left(\mathrm{cmol}_{\mathrm{c}} \mathrm{dm}^{-3}\right)$ & 1,85 & Densidade do solo $\left(\mathrm{g} \mathrm{cm}^{-3}\right)$ & 1,58 & $\mathrm{Fe}\left(\mathrm{mg} \mathrm{kg}^{-1}\right)$ & 149,8 & $1.705,5$ \\
\hline $\mathrm{Al}^{3+}\left(\mathrm{cmol}_{\mathrm{c}} \mathrm{dm}^{-3}\right)$ & 0,0 & Densidade de partícula $\left(\mathrm{g} \mathrm{cm}^{-3}\right)$ & 2,64 & $\mathrm{Zn}\left(\mathrm{mg} \mathrm{kg}^{-1}\right)$ & 55,20 & 144,5 \\
\hline $\operatorname{CTC}\left(\mathrm{cmol}_{\mathrm{c}} \mathrm{dm}^{-3}\right)$ & 4,26 & Porosidade total $\left(\mathrm{m}^{3} \mathrm{~m}^{-3}\right)$ & 0,40 & $\operatorname{Mn}\left(\mathrm{mg} \mathrm{kg}^{-1}\right)$ & 47,80 & 124,3 \\
\hline $\mathrm{V}(\%)$ & 56,3 & Microporosidade(\%) & 16,3 & $\mathrm{~B}\left(\mathrm{mg} \mathrm{kg}^{-1}\right)$ & 25,46 & 20,2 \\
\hline $\operatorname{MOS}\left(\mathrm{g} \mathrm{kg}^{-1}\right)$ & 13,1 & Macroporosidade (\%) & 83,7 & & & \\
\hline $\mathrm{S}\left(\mathrm{mg} \mathrm{dm}^{-3}\right)$ & 7,20 & Umidade - 0,010 MPa $\left(\mathrm{g} \mathrm{kg}^{-1}\right)$ & 64,8 & & & \\
\hline $\mathrm{B}\left(\mathrm{mg} \mathrm{dm} \mathrm{m}^{-3}\right)$ & 0,19 & Umidade- 0,033 MPa $\left(\mathrm{g} \mathrm{kg}^{-1}\right)$ & 48,0 & & & \\
\hline $\mathrm{Cu}\left(\mathrm{mg} \mathrm{dm}^{-3}\right)$ & 0,80 & Umidade - 1,500 MPa $\left(\mathrm{g} \mathrm{kg}^{-1}\right)$ & 25,1 & & & \\
\hline $\operatorname{Mn}\left(\mathrm{mg} \mathrm{dm}^{-3}\right)$ & 7,40 & Água disponível $\left(\mathrm{g} \mathrm{kg}^{-1}\right)$ & 39,8 & & & \\
\hline $\mathrm{Fe}\left(\mathrm{mg} \mathrm{dm}^{-3}\right)$ & 54 & & & & & \\
\hline $\mathrm{Zn}\left(\mathrm{mg} \mathrm{dm}^{-3}\right)$ & 1,84 & & & & & \\
\hline
\end{tabular}

$\mathrm{P}, \mathrm{K}^{+}$, e $\mathrm{Na}^{+}=$Extrator Melihch-1; $\mathrm{Al}^{3+}, \mathrm{Ca}^{2+}, \mathrm{Mg}^{2+}=$ Extrator $\mathrm{KCl} 1 \mathrm{M} ; \mathrm{SB}=$ Soma de bases trocáveis $\left(\mathrm{Ca}^{2+}+\mathrm{Mg}^{2+}+\mathrm{K}^{+}+\mathrm{Na}^{+}\right) ; \mathrm{CTC}^{2}$ Capacidade de troca catiônica $\left[\mathrm{SB}=\left(\mathrm{H}^{+}+\mathrm{Al}^{3+)}\right] ; \mathrm{V}=\right.$ Percentagem de saturação por bases $(\mathrm{SB} / \mathrm{CTC})$ 100; MOS = Matéria orgânica do solo. 


\section{RESULTADOS E DISCUSSÃO}

A interação potássio $\times$ cálcio não influenciou os teores foliares dos macronutrientes, exceto os de $\mathrm{S}(\mathrm{p} \geq$ 0,05). Quando aplicado de forma isolada, o $\mathrm{K}_{2} \mathrm{O}$ influenciou apenas os teores de $\mathrm{S}$, já o nitrato de cálcio influenciou os teores de P, K, Ca e S ( $\mathrm{p}<0,05$, Tabela 2). Considerando-se que o teor de $\mathrm{K}$ no solo é médio, esperavase que ao menos o teor foliar de $\mathrm{K}$ fosse influenciado pelas doses do nutriente, fato constatado em outros trabalhos a partir do segundo ciclo produtivo (Natale et al., 1996b; Amorim et al., 2015b). Quanto à influência do cálcio no teor dos nutrientes do tecido foliar, isso devese ao seu importante papel no crescimento do sistema radicular das plantas, contribuindo para maior absorção de nutrientes (Marschner, 2012) e, consequentemente, aumento do teor foliar.
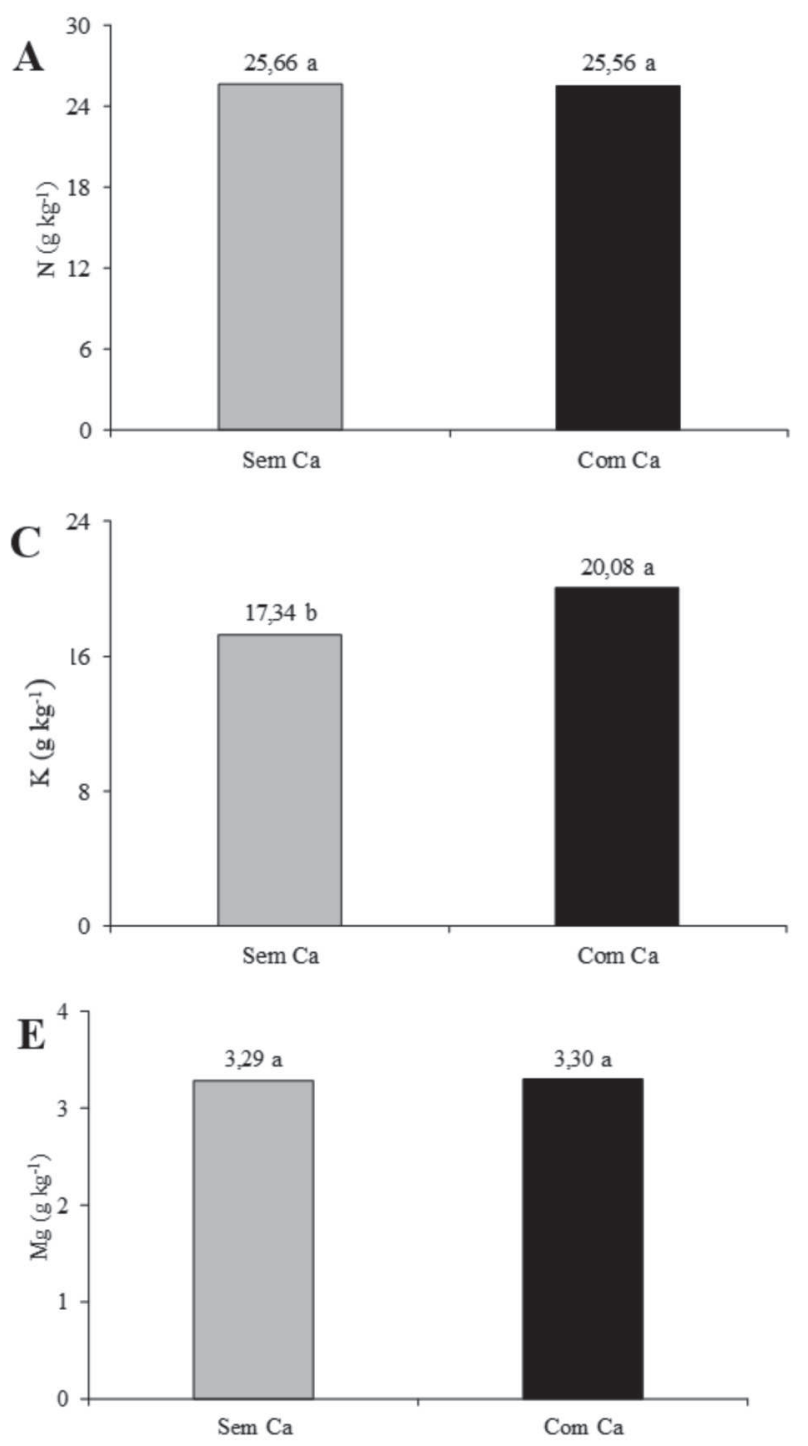

Os teores foliares de $\mathrm{N}$ nas plantas de goiabeira, apesar de não variarem com as doses de $\mathrm{K}_{2} \mathrm{O}$, foram de 25,7 e $25,6 \mathrm{~g} \mathrm{~kg}^{-1}$ no solo sem e com nitrato de cálcio, respectivamente (Figura 1A). Esses valores são superiores aos da faixa considerada adequada para a goiabeira 'Paluma', de 20 a $23 \mathrm{~g} \mathrm{~kg}^{-1}$ (Natale et al., 2009b). Esse resultado difere do obtido por Mikami et al. (2000) que constaram que a adubação com $\mathrm{K}$ e Ca proporcionou aumento do teor foliar de $\mathrm{N}$ em plantas de goiabeira 'Paluma' no estádio de pleno florescimento.

Os teores foliares de $\mathrm{P}$ aumentaram de 2,14 para 2,41 g $\mathrm{kg}^{-1}$, com a aplicação de cálcio (Figura 1B), e são superiores aos da faixa de 1,4 a 1,8 $\mathrm{g} \mathrm{kg}^{-1}$, adequada para a cultura (Natale et al., 2009b). Essa superioridade pode ser resposta da ação positiva do cálcio em estimular o crescimento radicular (Malavolta et al., 1997), aumentando a área de contato entre as raízes e o solo, proporcionando
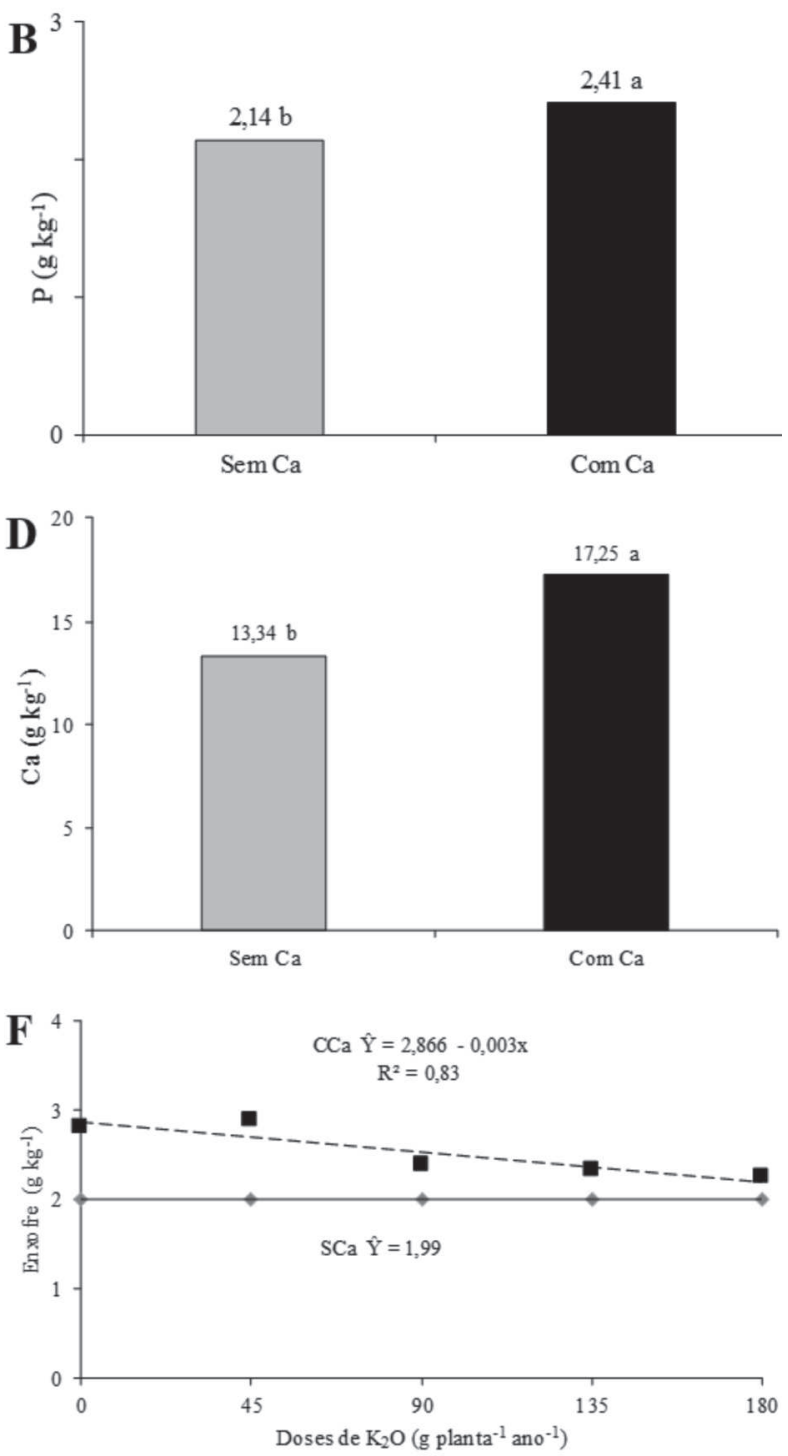

Figura 1: Teores foliares de nitrogênio (A), fósforo (B), potássio (C), cálcio (D), magnésio (E) e enxofre (F), em goiabeira 'Paluma', em função de doses de potássio, nos solos sem e com cálcio. 
maior absorção de nutrientes, em especial nutrientes de baixa mobilidade como o fósforo.

Os teores de $\mathrm{K}$ na matéria seca foliar da goiabeira foram de 17,3 e 20,1 $\mathrm{g} \mathrm{kg}^{-1}$ nas plantas não adubadas e adubadas com cálcio, respectivamente (Figura 1C). Em ambos os casos, os valores estão acima da faixa de exigência da cultura, que é de 14 a $17 \mathrm{~g} \mathrm{~kg}^{-1}$ (Natale et al., 2009b). Esse aumento dos teores foliares de $\mathrm{K}$ indica que a aplicação de $120 \mathrm{~g}$ planta $^{-1}$ ano $^{-1}$ de cálcio não inibiu a absorção de K pelas plantas. De acordo com Malavolta et al. (1997), a inibição competitiva entre $\mathrm{K}$ e Ca só ocorre quando este último encontra-se em alta concentração no solo. Entretanto, quando o Ca está em concentrações não muito elevadas no solo, aumenta a absorção de cátions como o K, por seu papel na manutenção da integridade funcional do plasmalema.

Ao se considerar que a nutrição das plantas com potássio, inclusive da goiabeira, ocorre em um sistema dinâmico pela alta mobilidade do nutriente no tecido vegetal (Hernandes et al., 2012; Sharma et al., 2012), os teores foliares do nutriente indicam suprimento adequado da cultura para produção de frutos (Amorim et al., 2015a). Esse suprimento adequado pode ser justificado pelo parcelamento da adubação potássica em três períodos, por causa da textura arenosa da área, permitindo maior disponibilidade do nutriente no solo e, consequentemente, maiores absorção e distribuição do K na planta (Zorb et al., 2014).

A aplicação de nitrato de cálcio ao solo refletiu-se em aumento do teor foliar de Ca nas plantas de goiabeira Paluma, com valores de 13,3 e 17,2 $\mathrm{g} \mathrm{kg}^{-1}$ (Figura 1D). Plantas de goiabeira do cultivar 'Paluma', segundo Natale et al. (2009b), quando adequadamente supridas de cálcio, devem apresentar teores foliares entre 7 e $15 \mathrm{~g} \mathrm{~kg}^{-1} \mathrm{e}$, de acordo com Souza et al. (2013), entre 8 e $11 \mathrm{~g} \mathrm{~kg}^{-1}$, determinados pelo método DRIS. Os valores indicam que a adubação com cálcio possivelmente proporcionou nutrição adequada das plantas, com resultados positivos no crescimento e na produção de frutos de goiabeira (Kumar et al., 2017).

Os teores foliares de $\mathrm{Mg}$, de forma semelhante ao verificado para $\mathrm{N}$, não variam com a interação potássio $\times$ cálcio, nem pela ação isolada de cada nutriente aplicado, apresentando o teor médio de 3,3 $\mathrm{g} \mathrm{kg}^{-1}$ (Figura 1E), abaixo do limite crítico inferior da variação de 3,4 a 4,0 $\mathrm{g} \mathrm{kg}^{-1}$ (Natale et al., 2009b), evidenciando que as plantas de goiabeira estavam nutricionalmente deficientes no macronutriente. Mikami et al. (2000) também não constataram influência da adubação com Ca e K sobre o teor foliar desses nutrientes, apesar de terem verificado aumento do teor de Ca e K no solo. Amorim et al. (2015b), avaliando a condição nutricional de plantios de goiabeira, por quantro ciclos consecutivos, observaram diminui- ção dos teores de $\mathrm{Mg}$ com o aumento das doses de $\mathrm{K}$ apenas nas avaliações realizadas no segundo e quarto ciclos.

Os teores foliares de $\mathrm{S}$ nas plantas não adubadas com nitrato de cálcio não se ajustaram a nenhum modelo de regressão utilizado e corresponderam ao valor médio de $1,99 \mathrm{~g} \mathrm{~kg}^{-1}$ (Figura 1F). Nas plantas adubadas com cálcio e potássio, os teores de $\mathrm{S}$ decresceram linearmente de 2,9 para 2,2 $\mathrm{g} \mathrm{kg}^{-1}$, entre as plantas sem e com $180 \mathrm{~g} \mathrm{planta}^{-1}$ $\mathrm{ano}^{-1}$ de $\mathrm{K}_{2} \mathrm{O}$. Independentemente da aplicação do nitrato de cálcio e das doses de $\mathrm{K}_{2} \mathrm{O}$, as plantas estavam deficientes em S, pois plantas de goiabeira 'Paluma' bem nutridas devem apresentar teores foliares de 2,5 a 3,5 $\mathrm{g} \mathrm{kg}^{-1}$ (Natale et al., 2009b).

Dentre os micronutrientes, apenas os teores foliares de boro, manganês e zinco foram influenciados pelo efeito isolado da adubação com nitrato de cálcio $(\mathrm{p}<0,05)$. Constata-se que não houve influência da interação potássio $\times$ cálcio nem das doses de $\mathrm{K}_{2} \mathrm{O}$ sobre o teor de nenhum dos micronutrientes avaliados $(\mathrm{p} \leq 0,05)$. Esses resultados divergem dos de Amorim et al. (2015b) que observaram aumento do teor de Mn com o aumento das doses de $\mathrm{K}_{2} \mathrm{O}$.

$\mathrm{O}$ teor foliar de $\mathrm{B}$ nas plantas de goiabeira aumentou de 82,5 para $196,2 \mathrm{mg} \mathrm{kg}^{-1}$ com a adubação de nitrato de cálcio (Figura 2A). O comportamento dos dados diverge dos de Dursun et al. (2010) que observaram efeito antagônico entre B e Ca, em hortaliças (tomate, pimentão e pepino). Esses valores dos teores foliares de B são marcadamente superiores aos das faixas de 20 a 25 e de 26 a $38 \mathrm{mg} \mathrm{kg}^{-1}$ admitida por Natale et al. (2009b) e por Souza et al. (2013) como adequadas para a goiabeira 'Paluma'. Esse incremento maior nos tratamentos com cálcio pode ser explicado pelas funções semelhantes do boro e do cálcio na síntese da parede celular (Malavolta et al., 1997).

Os teores foliares de $\mathrm{Cu}$ na goiabeira 'Paluma' não foram influenciados pelo potássio, pelo cálcio nem pela interação entre eles (Figura 2B). A goiabeira nutricionalmente suprida de $\mathrm{Cu}$ deve apresentar teores foliares entre 20 e $40 \mathrm{mg} \mathrm{kg}^{-1}$ (Natale et al., 2009b). Com base nas variações de 4,5 a 4,6 mg kg-1 , constata-se que as plantas, na época amostrada, estavam deficientes desse micronutriente (Figura 2B).

Os teores foliares de Fe na goiabeira 'Paluma' não foram influenciados pelo potássio nem pelo cálcio (Figura 2C). Plantas de goiabeira nutridas adequadamente de Fe devem apresentar teores na faixa de 60 a $90 \mathrm{mg} \mathrm{kg}^{-}$ ${ }^{1}$ (Natale et al., 2009b). Com base nas variações de 76,75 a $80,82 \mathrm{mg} \mathrm{kg}^{-1}$, constata-se que a goiabeira, na época amostrada, estava nutricionalmente equilibrada em ferro (Figura 2C).

As plantas de goiabeira apresentam aumento dos teores foliares de Mn de 51,5 para 60,8 $\mathrm{mg} \mathrm{kg}^{-1}$, nos tratamen- 
tos sem e com adubação com nitrato de cálcio, respectivamente (Figura 2D). Apesar de o Ca apresentar raio iônico semelhante ao do Mn, podendo inibir sua absorção e transporte (Marschner, 2012), esse fato não foi observado neste trabalho. Independentemente da adubação com cálcio, os teores situam-se na faixa de 40 a $80 \mathrm{mg} \mathrm{kg}^{-1}$, indicando que as plantas estavam nutridas com manganês (Natale $e t$ al., 1996a).

Os teores foliares de $\mathrm{Zn}$ foram de 20,7 e $22,3 \mathrm{mg} \mathrm{kg}^{-1}$ nas plantas sem e com adubação com nitrato de cálcio, respectivamente (Figura 2E). Isso justifica-se, por causa de o $\mathrm{Ca}$, em baixas concentrações, proporcionar maior absorção de Zn (Malavolta et al., 1997). Apesar do aumento proporcionado pelo $\mathrm{Ca}$, os valores, nos dois casos, estão abaixo da exigência da cultura, que oscila de 25 a $35 \mathrm{mg} \mathrm{kg}^{-1}$ (Natale et al., 1996a) e evidenciam que as plantas estavam deficientes do micronutriente.
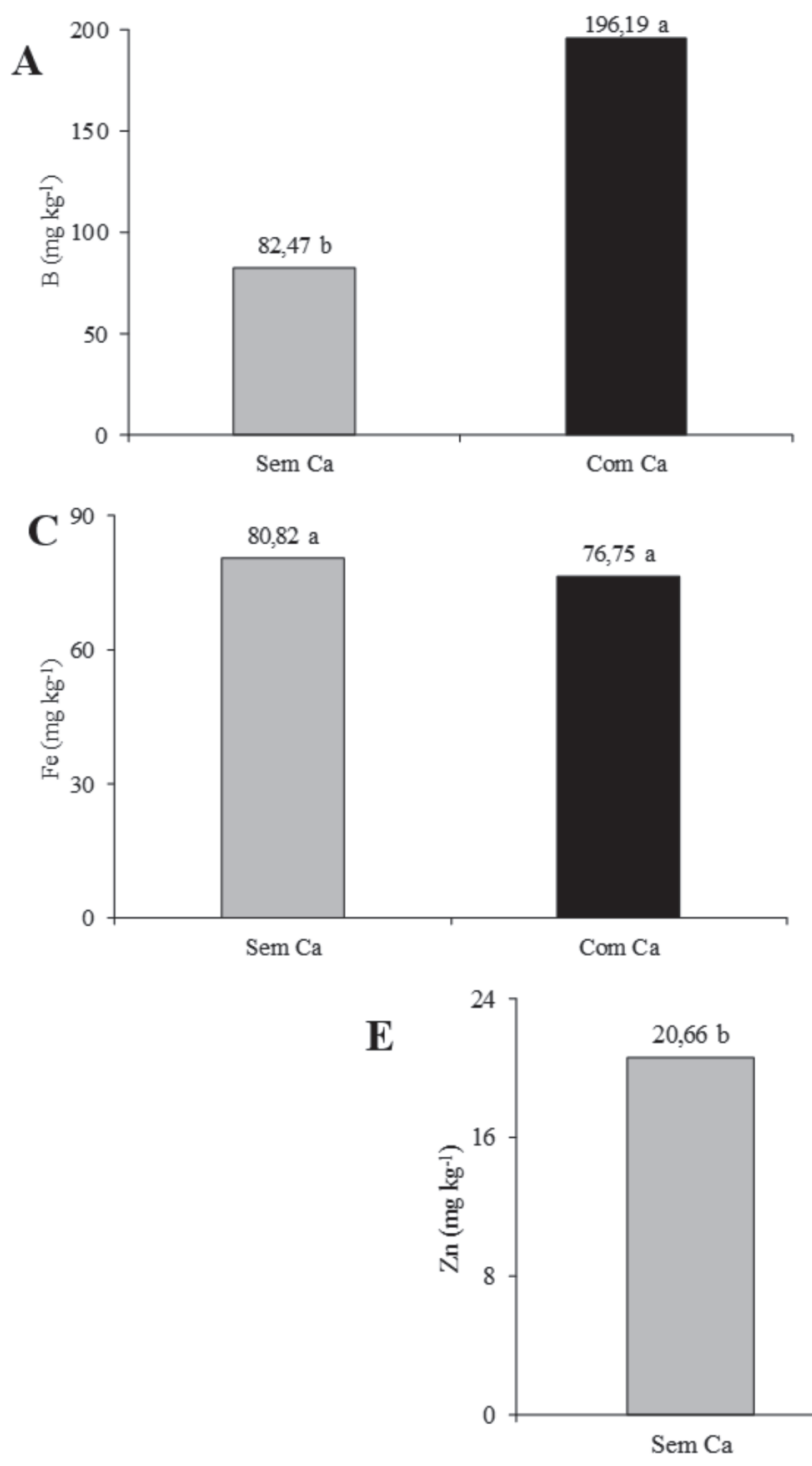

Pelos resultados, constata-se que a adição de $20 \mathrm{~g}$ por planta de sulfato de zinco, antes da aplicação dos tratamentos, e a liberação de 55,20 a 144,25 mg kg-1 (Tabela 1), por mineralização da matéria orgânica da cobertura morta (Teixeira et al., 2003b; Moreira et al., 2016; Moreira et al., 2017), não foram suficientes para disponibilizar $\mathrm{Zn}$ em quantidades adequadas para suprir a exigência das plantas. Essa deficiência de Zn pode ter sido induzida pelos elevados teores de Fe presentes no solo (Tabela 1), pelo fato de o Fe inibir a absorção de Zn (Malavolta et al., 1997).

O aumento das doses de potássio de 0 até as máximas estimadas de 158 e 114 g planta $^{-1}$ ano $^{-1}$ de $\mathrm{K}_{2} \mathrm{O}$ elevou o número de frutos, colhidos por planta, de 82,5 e 88,1 para 182 e 140 frutos nas plantas sem e com adubação de nitrato de cálcio, respectivamente (Figura 3A). Esses valores estão muito aquém dos 232 frutos planta ${ }^{-1}$ obtidos com a
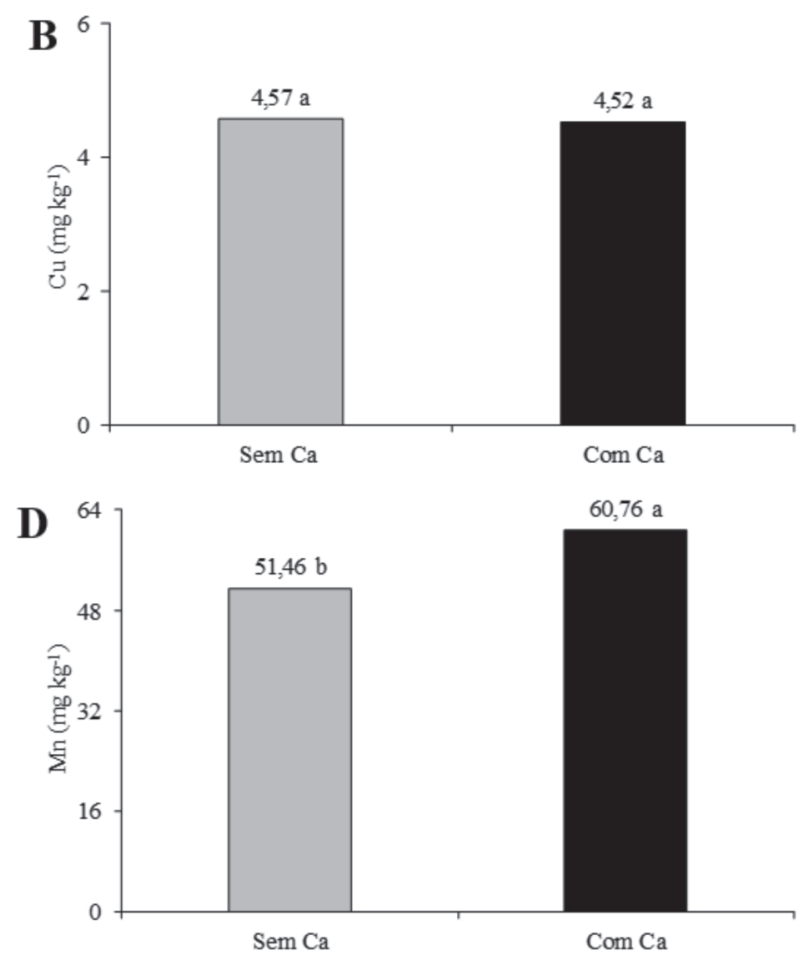

22,27 a

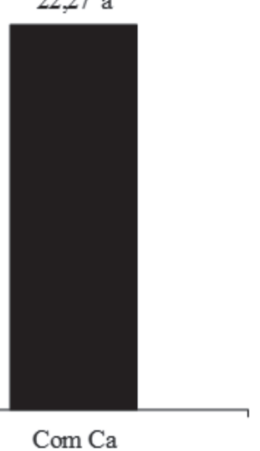

Figura 2: Teores foliares de boro (A), cobre (B), ferro (C), manganês (D) e zinco (E), em goiabeira 'Paluma', nos solos sem e com cálcio. 
dose de $2 \mathrm{~kg}$ planta ${ }^{-1}$ de $\mathrm{K}_{2} \mathrm{O}$ por Alencar et al. (2016), utilizando o mesmo cultivar de goiaba, em plantas com três anos de idade. Entretanto, as plantas do presente trabalho tinham 22 meses de idade, o que pode justificar essa diferença na produção de frutos.

$\mathrm{O}$ aumento das doses de $\mathrm{K}$ acima das máximas estimadas até a dose de $180 \mathrm{~g}_{\text {planta }}{ }^{-1} \mathrm{ano}^{-1}$ de $\mathrm{K}_{2} \mathrm{O}$ reduziu o número de frutos planta ${ }^{-1}$ de 182 para 179 e de 140 para 122, nas plantas não adubadas e nas adubadas com nitrato de cálcio, respectivamente, indicando que doses elevadas de $\mathrm{K}_{2} \mathrm{O}$ comprometeram a produção de frutos da goiabeira. Essa redução do número de frutos ocorre pelo fato de o $\mathrm{K}$ em concentrações elevadas no solo, inibir a absorção de $\mathrm{Ca}$ e $\mathrm{Mg}$, comprometendo funções fisiológicas importantes das plantas (Marschner, 2012).

Nas plantas não adubadas com cálcio, o aumento das doses de potássio, com pontos de máximo atingidos na dose estimada de $101 \mathrm{~g}_{\text {planta }}{ }^{-1} \mathrm{ano}^{-1}$ de $\mathrm{K}_{2} \mathrm{O}$, elevou a massa média de frutos até $173,3 \mathrm{~g}$ fruto $^{-1}$ (Figura 3B). Não se obteve ajuste de modelo para essa variável nas plantas adubadas com cálcio, com média de $172,7 \mathrm{~g}_{\text {fruto }}{ }^{-1}$. Esses resultados estão de acordo com os de Natale et al. (1996b) que observaram aumento da massa média de frutos em função da adubação potássica. Por outro lado, Amorim et al. (2015a), em plantas de goiabeira 'Paluma' com sete anos de idade, não verificaram efeito da adubação potássica sobre a massa média de frutos, com valores de 117,147 e $168 \mathrm{~g}$ frutos $^{-1}$, no primeiro, segundo e terceiro
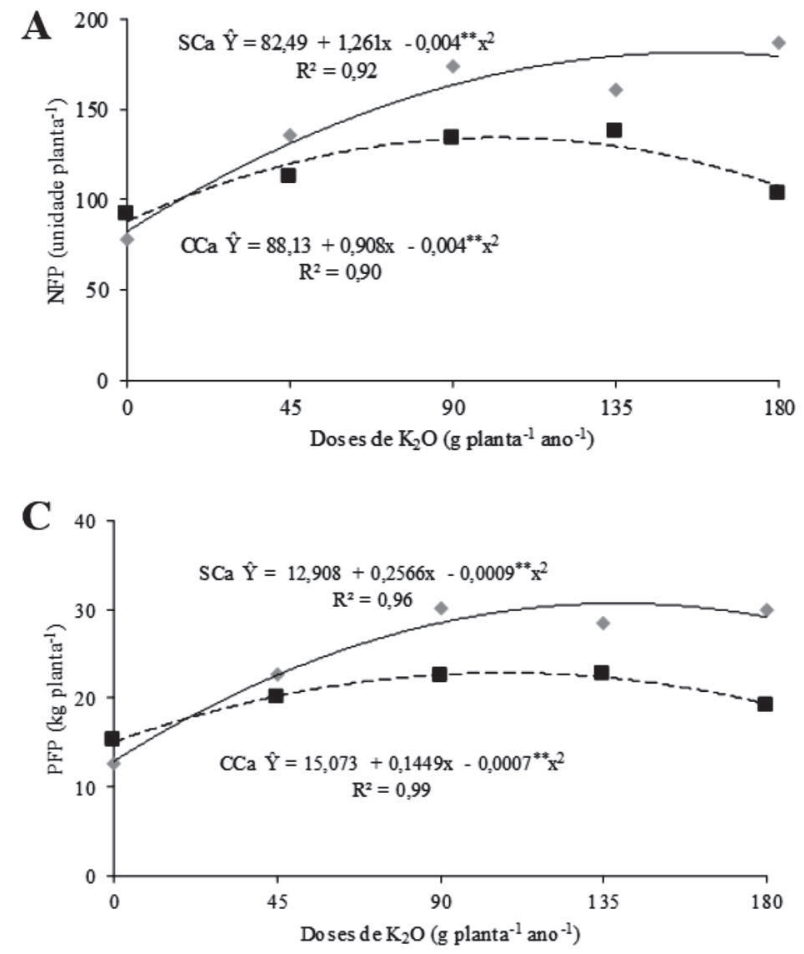

ciclo. Isso indica que a adubação com cálcio proporcionou nutrição adequada das plantas, justificando o incremento da massa média de frutos de goiabeira (Jayachandra et al., 2005; Kumar et al., 2017).

O aumento das doses de potássio, até as máximas estimadas de 143 e $104 \mathrm{~g} \mathrm{planta}^{-1} \mathrm{ano}^{-1}$, elevou a produção por planta para valores de 31,2 e 25,6 kg planta ${ }^{-1}$, no solo sem e com nitrato de cálcio, respectivamente (Figura 3C). Entretanto, a adubação com doses de $\mathrm{K}_{2} \mathrm{O}$ acima das máximas estimadas inibiu a capacidade produtiva da goiabeira 'Paluma', em ambas as situações, por causa de o K induzir deficiência de $\mathrm{Ca}$ e $\mathrm{Mg}$ nas plantas, quando em concentrações elevadas no solo (Malavolta et al., 1997; Marschner, 2012). Esses valores estão bem abaixo dos $55,7 \mathrm{~kg}$ planta $^{-1}$ produzidos com a dose de $330 \mathrm{~g} \mathrm{planta}^{-1}$ $\mathrm{ano}^{-1}$ de $\mathrm{K}_{2} \mathrm{O}$, na primeira safra, em goiabeira 'Paluma' irrigada (Alencar et al., 2016) e assemelham-se aos $25 \mathrm{~kg}$ planta $^{-1}$ de frutos de goiaba na dose $400 \mathrm{~g} \mathrm{planta}^{-1} \mathrm{de}_{2} \mathrm{O}$ (Lima et al., 2008). Esses resultados corroboram os de Souza et al. (2012) e Souza et al. (2014) ao concluírem que a goiabeira é muito exigente em $\mathrm{K}$, por isso, a adubação com esse macronutriente justifica seu efeito positivo no crescimento vegetativo e na produção de frutos.

Apesar da diminuição de produção provocada pelo nitrato de cálcio, em relação à das plantas sem o fertilizante, a produção por planta supera os $15,2 \mathrm{~kg}$ planta $^{-1}$ colhidos em goiabeira 'Paluma' com 20 meses de idade, cultivada na mesma localidade deste trabalho (Rocha et al., 2016).
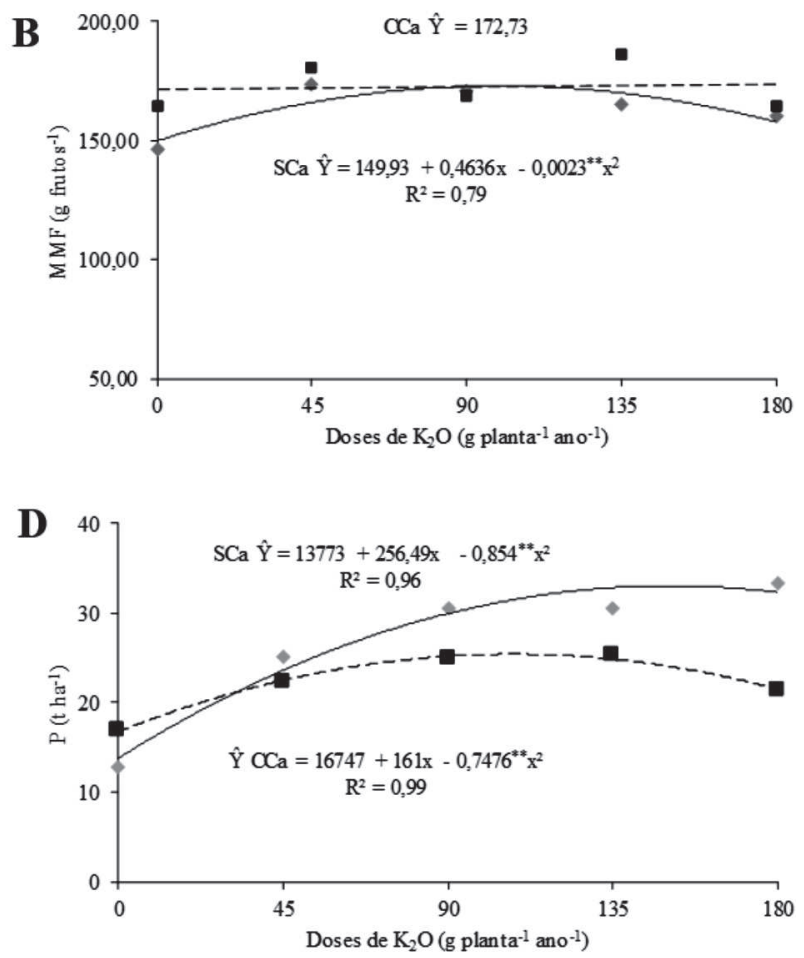

Figura 3: Número de frutos por planta - NFP (A), massa média de frutos - MMF (B), produção de frutos por planta - PFP (C) e produtividade - P (D), em goiabeira 'Paluma', em função de doses de potássio, nos solos sem cálcio (SCa) e com cálcio (CCa). 
Possivelmente, o excesso de $\mathrm{K}$ aplicado nos tratamentos das maiores doses pode ter causado um desbalanço nutricional entre o $\mathrm{K}$ e outros nutrientes, contribuindo para a redução do crescimento e, consequentemente, para a redução de produção da cultura (Andrade Junior et al., 2005). Para realização da adubação potássica deve-se considerar o equilíbrio entre o $\mathrm{K}$ e cátions como $\mathrm{Ca}$ e $\mathrm{Mg}$, pois cátions monovalentes são absorvidos pelas raízes das plantas mais rapidamente que os bivalentes; assim, uma relação adequada entre eles é fundamental para aumentar a eficiência de absorção (Montes et al., 2016).

A produtividade de frutos aumentou com as doses de $\mathrm{K}_{2} \mathrm{O}$ aplicadas, porém, a maior eficiência do fertilizante potássico foi observada nos tratamentos sem aplicação de cálcio (Figura 3D). Nas plantas não adubadas com nitrato de cálcio, na dose máxima estimada de $143 \mathrm{~g}$ planta${ }^{1}$ ano $^{-1}$ de $\mathrm{K}_{2} \mathrm{O}$, a produtividade foi de $19.130,8 \mathrm{~kg} \mathrm{ha}^{-1}$. Esses resultados diferem dos de Natale et al. (1996b) que não evidenciaram resposta de doses de até $0,6 \mathrm{~kg}$ planta${ }^{1}$ de $\mathrm{K}_{2} \mathrm{O}$ no primeiro e segundo ciclo da cultura, e dos de Amorim et al. (2015a), que não constataram influência da adubação potássica em plantas de goiabeira com sete anos de idade, com doses de até 2,2 $\mathrm{kg}_{\text {planta }}{ }^{-1}$ de $\mathrm{K}_{2} \mathrm{O}$.

Quando o K foi aplicado em doses elevadas, a produtividade de frutos de goiabeira 'Paluma' foi prejudicada, principalmente nas plantas nas quais foi aplicado o nitrato de cálcio. De acordo com Marschner (2012), cátions como o K podem atravessar a membrana plasmática com maior velocidade, deprimindo a absorção de cátions mais lentos como $\mathrm{Ca}$ e $\mathrm{Mg}$, podendo causar deficiência nas plantas e comprometendo a produtividade.

A aplicação de fertilizantes potássicos, na dose necessária para a melhoria da fertilidade do solo e para atender a necessidade nutricional da cultura, proporciona maior incremento da produtividade agrícola (Feitosa et al., 2013). Porém, é importante destacar que a utilização de altas doses de cloreto de potássio pode causar toxidade nas plantas (Miyake et al., 2016) e perdas fotossintéticas na goiabeira (Cavalcante et al., 2018) e esses efeitos provocam perdas de produtividade da cultura.

\section{CONCLUSÕES}

A adubação potássica, aplicada juntamente com o Ca, não altera os teores foliares de macro e micronutrientes, exceto do enxofre que foi reduzido com o aumento das doses.

A adubação com cálcio promove aumento dos teores foliares de fósforo, potássio, cálcio, enxofre, boro, manganês e zinco.

A adição de cálcio juntamente com a adubação potássica reduziu a capacidade produtiva das plantas.
A máxima capacidade produtiva das plantas de goiabeira Paluma foi obtida sob adubação potássica, com doses variando entre 101 e $143 \mathrm{~g}$ planta $^{-1}$ ano $^{-1} \mathrm{~K}_{2} \mathrm{O}$, sem aplicação de cálcio.

\section{AGRADECIMENTOS}

Os autores agradecem à Coordenação de Aperfeiçoamento de Pessoal de Nível Superior (CAPES), pela concessão da bolsa de mestrado ao primeiro autor, e ao Conselho Nacional de Desenvolvimento Científico e Tecnológico (CNPq), pela contribuição financeira ao trabalho. Agradecem também ao Programa de Pós-Graduação em Agronomia da Universidade Federal da Paraíba pelo apoio no desenvolvimento da pesquisa.

\section{REFERÊNCIAS}

Alvares CA, Stape JL, Sentelhas PC, Gonçalves JLM \& Sparovek G (2013) Köppen's climate classification map for Brazil. Meteorologische Zeitschrift, 22:711-728.

Alencar RD, Leite GA, Mendonça V, Lima FV, Pereira GA \& Farias WC (2016) Adubação potássica na produção e qualidade pós-colheita de goiaba 'Paluma' no semiárido potiguar. Comunicata Scientiae, 07:139-148.

Amorim DA, Rozane DE, Souza HA, Modesto VC \& Natale W (2015a) Adubação nitrogenada e potássica em goiabeiras 'Paluma': I. Efeito na produtividade e na qualidade dos frutos para industrialização. Revista Brasileira de Fruticultura, 37:201209.

Amorim DA, Souza HA, Rozane DE, Montes RM \& Natale W (2015b) Adubação nitrogenada e potássica em goiabeiras 'Paluma':II. Efeito no estado nutricional das plantas. Revista Brasileira de Fruticultura, 37:210-219.

Andrade Junior AS, Dias NS, Figueredo Junior LGM, Daniel R \& Ribeiro VQ (2005) Doses de potássio via fertirrigação na produção e qualidade de frutos de melancia em Parnaíba, PI. Irriga, 10:205-214

Aular J \&, Natale W (2013) Nutrição mineral e qualidade do fruto de algumas frutíferas tropicais: goiabeira, mangueira, bananeira e mamoeiro. Revista Brasileira de Fruticultura, 35:1214-1231.

Dias MJT, Souza HA, Natale W, Modesto VC \& Rozane DE (2012) Adubação com nitrogênio e potássio em mudas de goiabeira em viveiro comercial. Semina: Ciências Agrárias, 33:2837-2848.

Cavalcante ACP, Cavalcante LF, Cavalcante AG, Bertino AMP, Bertino AMP \& Ferreira NM (2018) Physiology of Paluma guava plants fertilized with potassium and calcium. Idesia, 36:163-172.

Cody R, (2015) An introduction to SAS University Edition. Cary, SAS Institute. 366p.

Dursun A, Turan M, Ekinci M, Gunes A, Ataoglu N, Esringu A \& Yildirim E (2010) Effects of boron fertilizer on tomato, pepper and cucumber yields and chemical composition. Communications in Soil Science Plant Analysis, 41:1576-1593.

Embrapa - Empresa Brasileira de Pesquisa Agropecuária (2013) Sistema Brasileiro de Classificação de Solos. $2^{\text {a }}$ ed. Rio de Janeiro, Embrapa Solos. 353p.

Embrapa - Empresa Brasileira de Pesquisa Agropecuária (2011) Manual de Métodos de Análise do Solo. $3^{\mathrm{a}}$ ed. Rio de Janeiro, Embrapa - CNPS. 230p. 
Embrapa - Empresa Brasileira de Pesquisa Agropecuária (2009) Manual de análises químicas de solos, plantas e fertilizantes. $2^{a}$ ed. Rio de Janeiro, Embrapa Solos. 627p.

Feitosa HO, Farias GC, Silva Junior RJC, Ferreira FJ, Andrade Filho FL \& Lacerda CF (2013) Influência da adubação borácica e potássica no desempenho do girassol. Comunicata Scientiae, 04:302-307.

França SC, Oliveira AC, Farias GA, Cabral Junior LF \& Silva VL (2017) Doses de nitrogênio no crescimento de porta-enxerto de goiabeira Paluma amarela. Scientia Agraria, 18:54-65.

Hernandes A, Parent SÉ, Natale W \& Parent LÉ (2012) Balancing guava nutrition with liming and fertilization. Revista Brasileira Fruticultura, 34:1224-1234.

Jayachandra KS, Srihari D \& Reddy YN (2005) Preharvest spray of different source of calcium to improve the shelf life of guava. Indian Journal of Horticultural Science, 62:68-70.

Karley AJ \& White JP (2009) Moving cationic minerals to edible tissues: potassium, magnesium, calcium. Current Opinion in Plant Biology, 12:291-298.

Kumar J, Kumar R, Rai R, Mishra DS, Singhl SK \& Nimbolkar PK (2017) Influence of foliar application of mineral nutrients at different growth stages of guava. Journal of Plant Nutrition, 40:656-661.

Lima MAC, Bassoi LH, Silva DJ, Santos PS, Paes PC, Ribeiro PRA \& Dantas BF (2008) Effects of levels of nitrogen and potassium on yield and fruit maturation of irrigated guava trees in the São Francisco valley. Revista Brasileira de Fruticultura, 30:246-250.

Malavolta E, Vitti GC \& Oliveira AS (1997) Avaliação do Estado Nutricional das Plantas. Princípios e aplicações. Piracicaba, Associação Brasileira para Pesquisa da Potassa e do Fosfato. 319 .

Marschner P (2012) Marchner's mineral nutrition of higher plants. $3^{\text {rd }}$ ed. New York, Academic Press. 651p.

Mikami EE, Pintro JC, Tormena CA, Costa ACS \& Sengik E (2000) Influência da aplicação de cálcio, de magnésio e de potássio no solo sobre a produção de goiaba (Psidium guajava L.) cv. Acta Scientiarum, 22:1075-1081.

Miyake RTM, Takata WHS, Guerra WEX, Forli F, Narita N \& Creste JE (2016) Effects of potassium fertilization and commercial substrates on development of passion fruit seedlings under greenhouse condition. African Journal of Agricultural Research, 11:3720-3727.

Montes RM, Parent LÉ, Amorim DA, Rozane DE, Parent SÉ, Natale W \& Modesto VC (2016) Nitrogen and potassium fertilization in a guava orchard evaluated for five cycles: Effects on the plant and on production. Revista Brasileira de Ciência do Solo, 40:01-12.

Moreira SG, Prochnow LI, Kiehl JC, Pauletti V \& Martin-Neto L (2016) Chemical forms in soil and availability of manganese and zinc to soybean in soil under different tillage systems. Soil \& Tillage Research, 163:41-53.
Moreira SG, Prochnow LI, Pauletti V, Silva BM, Kiehl JC \& Silva CGM (2017) Effect of liming on micronutrient availability to soybean grown in soil under different lengths of time under no tillage. Acta Scientiarum Agronomy, 39:89-97.

Natale W, Rozane DE, Souza HA \& Amorim DA (2009a) Cultura da goiaba: do plantio a comercialização. Jaboticabal, Funep. 284 p.

Natale W, Prado RM, Quaggio JA \& Mattos Júnior D (2009b) Goiabeira. In: Crisósto MO LA \& Naumov A (Ed.) Adubando para alta produtividade e qualidade: fruteiras tropicais do Brasil. Fortaleza, Embrapa Agroindústria Tropical. p.104-124.

Natale W, Coutinho ELM, Boaretto AE \& Pereira FM (1996a) Goiabeira: calagem e adubação. Jaboticabal, Funep. 22p.

Natale W, Coutinho ELM, Boaretto AE, Pereira FM, Oioli AAP \& Sales L (1996b) Nutrição e adubação potássica na cultura da goiabeira. Revista Brasileira de Ciência do Solo, 20:247-250.

Natale W, Prado RM \& Moro FV (2005) Alterações anatômicas induzidas pelo cálcio na parede celular de frutos de goiabeira. Pesquisa Agropecuária Brasileira, 40:1239-1242.

Rocha LF, Cavalcante LF, Nunes JC, Souto AGL, Cavalcante ACP, Cavalcante ÍHL \& Pereira WE (2016) Fruit production and quality of guava 'Paluma' as a function of humic substances and soil mulching. African Journal of Biotechnology, 15:1962-1969.

Sharma V, Sharma S, Arora S \& Kumar A (2012) Quantity-intensity relationships of potassium in soils under some guava orchards on marginal lands. Communications in Soil Science and Plant Analysis, 43:1550-1562.

Silva RP, Peixoto JR \& Junqueira NTV (2001) Influência de diversos substratos no desenvolvimento de mudas de maracujazeiro azedo (Passiflora edulis Sims f. flavicarpa Deg). Revista Brasileira de Fruticultura, 23:01-05.

Souza HA, Rozane DE, Romualdo LM \& Natale W (2012) Efeitos de diferentes tipos de poda nos teores de nutrientes em flores e frutos de goiabeira. Idesia, 30:45-51.

Souza HA, Rozane DE, Amorim DA \& Natale W (2013) Normas preliminares DRIS e faixas de suficiência para goiabeira 'Paluma'. Revista Brasileira de Fruticultura, 35:282-291.

Souza HA, Rozane DE, Amorim DA, Modesto VC \& Natale W (2014) Uso fertilizante do subproduto da agroindústria processadora de goiabas I - Atributos químicos do solo. Revista Brasileira de Fruticultura, 36:713-724.

Teixeira AHC, Bassoi LH, Reis VCS, Silva TGF, Ferreira MNL \& Maia JLT (2003a) Estimativa do consumo hídrico da goiabeira, utilizando estações agrometeorológicas automática e convencional. Revista Brasileira de Fruticultura, 25:457-460.

Teixeira IR, Souza CM, Borém A \& Silva GF (2003b) Variação dos valores de $\mathrm{pH}$ e dos teores de carbono orgânico, cobre, manganês, zinco e ferro em profundidade em argisssolo vermelho-amarelo, sob diferentes sistemas de preparo do solo. Bragantia, 62:119-126.

Zorb C, Senbayram M \& Peiter E (2014) Potassium in agriculturestatus and perspectives. Journal of Plant Physiology, 171:656669. 